\title{
Excitation of Multiple Spatial Modes of a MEMS Cantilever in the Pulsed Digital Oscillator
}

\author{
Elena Blokhina and Orla Feely \\ School of Electrical, Electronic and Mechanical Engineering \\ University College Dublin \\ Dublin, Ireland
}

\author{
Manuel Domínguez \\ Micro and Nano Technologies group \\ Universitat Politècnica de Catalunya \\ Barcelona, Spain
}

\begin{abstract}
The aim of this paper is to apply an approach that will allow us to consider different mechanical modes of a MEMS cantilever in the form of separate mass-spring-damper equations with the appropriate form of an external driving. In the paper, we focus on a specific MEMS cantilever and use a Pulsed Digital Oscillator (PDO) to keep self-sustained oscillations of the mechanical structure. By applying the order-reduction procedure to a partial differential equation that describes the transversal deflections, we obtain a system of coupled ordinary differential equations that describes the excitation of multiple spatial modes. On the basis of these ordinary differential equations, we formulate a set of iterative maps to describe the evolution of the modes between two sampling events. The numerical simulations we present are focused on the most common case when the first two mechanical modes are taken into consideration.
\end{abstract}

\section{INTRODUCTION}

Resonant sensors exploit mechanical structures that are excited in one of their mechanical modes. These mechanical structures can be beams [1]-[3] or plates (membranes) [4] with additional supporting elements (springs, arms, etc.). Typically, a plate that is clamped across one of its edges (usually at the short end(s)) displays predominantly one-dimensional oscillations and can be modelled by using equations for elastic beams.

The dynamics of continuous structures in MEMS are described by partial differential equations (PDEs) with appropriate boundary conditions. The most typical way to deal with the problem is to reduce distributed systems in the form of PDEs to lumped systems in the form of ordinary differential equations (ODEs). These ODEs usually take the form of the mass-spring-damper equation. Linear or nonlinear massspring-damper equations are a typical and yet effective way to model the dynamics of a variety of MEMS systems [4]-[7].

There are a number of procedures that allow us to develop the reduced-order models from the original PDEs (see [8], [9] and the references cited there). A wide class of methods is based on eliminating the spatial dependencies in the PDEs by means of a set of spatial eigenmode functions $\psi_{i}(x, y, z)$, which in a general case is infinite. As a result, we will obtain a set of ordinary differential equations that represent the dynamics of each spatial mode as the mass-spring-damper equation with appropriate parameters.

In this paper, we focus on a specific type of resonant MEMS device embedded inside a pulsed digital oscillator (PDO). The

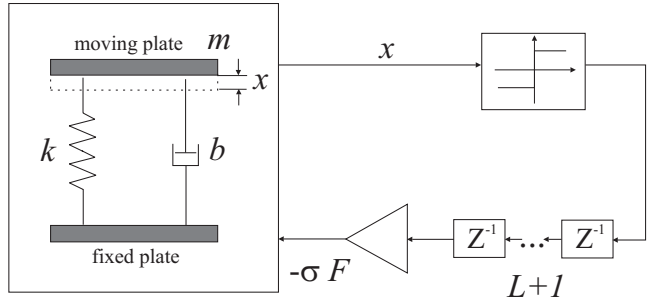

Fig. 1. General single feedback topology of the pulsed digital oscillator. $L+1$ is the number of delay blocks and $\sigma$ is the sign of the feedback loop.

PDO is a large signal oscillator that utilises a micromechanical resonator in a feedback loop of a type well known from the domain of sigma-delta modulation. The general singlefeedback topology of this oscillator is shown in Fig. 1. The position of the resonator is evaluated at each sampling time, and short force pulses (which can take two possible values depending on the position of the resonator) are applied to actuate the resonator. The output of the system is the bitstream provided by the 1-bit quantizer.

We start with the partial differential equation that describes the transversal deflections of a beam [10]. This PDE provides a more general description than a model based only on massspring-damper equations. We apply the procedure based on presenting a solution using linear mode shapes. As a result, we obtain a system of ordinary differential equations that are coupled via the external driving. We formulate a set of iterative equations (maps) to describe the evolution of the spatial modes between two sampling events. In this paper, we focus mostly on the simplest case when only the first two mechanical modes are taken into consideration. We present results of numerical simulations and show that depending on values of the sampling frequency, the system displays oscillations that can be associated with various spatial mode.

\section{Statement of The PROBlem}

In this section we focus on deriving the reduced-order model from the partial differential equation. Let us assume that the MEMS we study utilises a clamped-free cantilever of the type shown in Fig. 2. We consider that the beam is excited by the external force $F(\xi, \tau)$. The equation and the boundary conditions that describe the transverse vibrations of the beam 


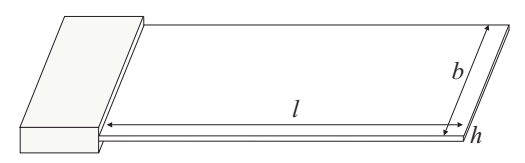

Fig. 2. A schematic drawing of the MEMS resonator: a clamped-free beam.

in the dimensionless form are as follows [10]:

$$
\begin{gathered}
\frac{\partial^{4} u}{\partial \xi^{4}}+\frac{\partial^{2} u}{\partial \tau^{2}}+\gamma \frac{\partial u}{\partial \tau}=F(\xi, \tau), \\
u(0, \tau)=\frac{\partial u(0, \tau)}{\partial \xi}=0, \quad \frac{\partial^{2} u(1, \tau)}{\partial \xi^{2}}=\frac{\partial^{3} u(1, \tau)}{\partial \xi^{3}}=0,
\end{gathered}
$$

with the dimensionless variables and parameters

$$
\xi=x / l, \quad \tau=\frac{t}{l^{2}} \sqrt{\frac{E I}{\rho A}}, \quad \gamma=\frac{c l^{2}}{\sqrt{\rho A E I}} .
$$

In (1), (2) and (3) $u(x, t)$ is the transverse displacement at the position $\xi$ and time $\tau, E$ is the Young's modulus, $I$ is the moment of inertia of the cross-section (for beams with a rectangular cross-section $\left.I=b h^{3} / 12\right), \rho$ is the density, $A$ is the area of the cross-section, $c$ is the dissipation coefficient, $\gamma$ is the dimensionless dissipation parameter, $l$ is the length of the beam, $b$ is its width and $h$ is its thickness.

Next, we discretise the continuous equation (1) by using a truncated set of linear mode shapes

$$
u(\xi, \tau)=\sum_{i=1}^{M} \xi_{i}(\tau) \psi_{i}(\xi)
$$

For a clamped-free beam, these functions are [10]

$$
\psi_{i}(\xi)=\cosh \left(\Omega_{i} \xi\right)-\cos \left(\Omega_{i} \xi\right)+\nu_{i}\left(\sin \left(\Omega_{i} \xi\right)-\sinh \left(\Omega_{i} \xi\right)\right)
$$

where $\nu_{i}=\left(\cos \Omega_{i}+\cosh \Omega_{i}\right) /\left(\sin \Omega_{i}+\sinh \Omega_{i}\right)$, and $\Omega_{i}$ are the roots of the equation $\cos \Omega \cosh \Omega=-1$. The first three spatial functions $\psi_{1,2,3}(\xi)$ as functions of the axial coordinate are shown in Fig. 3. Later in the paper, we will refer to vibrations that correspond to these functions as spatial modes with the spatial frequencies $\Omega_{i}$ and the temporal frequencies $\Omega_{i}^{2}$.

Substituting (4) into (1), multiplying the latter equation by $\psi_{j}$, integrating over the beam domain and taking into account the orthogonality of the functions $\psi_{j}$, we obtain the system of ordinary differential equations

$$
\ddot{x}_{i}+\gamma \dot{x}_{i}+\Omega_{i}^{4} x_{i}=F_{i}(\tau), \quad i=\overline{1, M} .
$$

Note that the system (6) presents each spatial mode as the mass-spring-damper system with appropriate parameters such as the dissipation parameter $\gamma$ and the temporal frequency $\omega_{0 i}=\Omega_{i}^{2}$.

The functions $F_{i}$ in the right hand of the equations are the 'projections' of the external force to each eigenmode. They

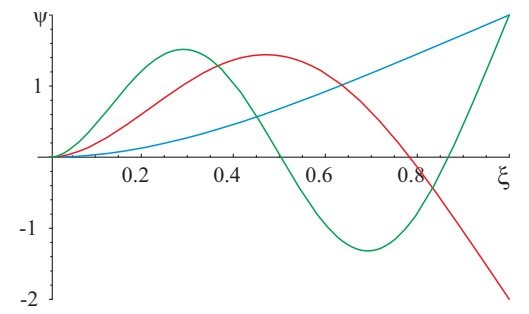

Fig. 3. Functions $\psi_{1,2,3}(\xi)$ versus the axial coordinate $\xi$.

define what quantity of the force is transmitted to excite the oscillations of a mode:

$$
F_{i}(\tau)=\int_{0}^{1} \psi_{i}(\xi) F(\xi, \tau) d \xi
$$

Now let us return to the PDO and rewrite the system of equations (6) for our specific case. The external driving has the following form:

$F(\xi, \tau)=-\sigma F_{0} \sum_{n} \operatorname{sign}\left(u\left(\xi_{s}, \tau_{n-L-1}\right)\right) \delta\left(\xi-\xi_{a}\right) \delta\left(\tau-\tau_{n}\right)$,

where $L+1$ is the number of delays in the feedback (see Fig. 1), $\tau_{n}=n T_{s}$ is the instants of time at which we apply the force pulse, $T_{s}=1 / f_{s}$ is the time between two impulses, $\delta(x)$ is the Dirac delta function and $\operatorname{sign}(x)$ is the signum function. In eq. (8), $\xi_{s}$ is the point of the microcantilever at which the sensing system measures the resonator position and $\xi_{a}$ is the point at which the actuating system applies the external driving in order to maintain the oscillations. If we restrict ourselves to the case of the first two spatial modes, the set of equations becomes

$$
\begin{aligned}
& \ddot{x_{1}}+\gamma \dot{x_{1}}+\Omega_{1}^{4} x_{1}=-\sigma F_{01} \sum_{n} b_{n-L-1} \delta\left(\tau-\tau_{n}\right), \\
& \ddot{x_{2}}+\gamma \dot{x_{2}}+\Omega_{2}^{4} x_{2}=-\sigma F_{02} \sum_{n} b_{n-L-1} \delta\left(\tau-\tau_{n}\right),
\end{aligned}
$$

where $b_{n}$ is the sequence of signs

$$
b_{n}=\operatorname{sign}\left\{x_{1}\left(\tau_{n}\right)+\beta x_{2}\left(\tau_{n}\right)\right\},
$$

the coefficients $\beta$ and $\mu$ describe the sensing and actuating systems

$$
\beta=\psi_{2}\left(\xi_{s}\right) / \psi_{1}\left(\xi_{s}\right), \quad \mu=\psi_{2}\left(\xi_{a}\right) / \psi_{1}\left(\xi_{a}\right)
$$

and $F_{0 i}$ are the amplitudes of the external driving for the $i$ th spatial mode in the corresponding equation

$$
F_{0 i}=F_{0} \psi_{i}\left(\xi_{a}\right)
$$

In this paper we assume that $\xi_{s}=\xi_{a}=1$, i.e. $\beta=\mu=-1$, and the sign of the feedback loop $\sigma=-1$. 

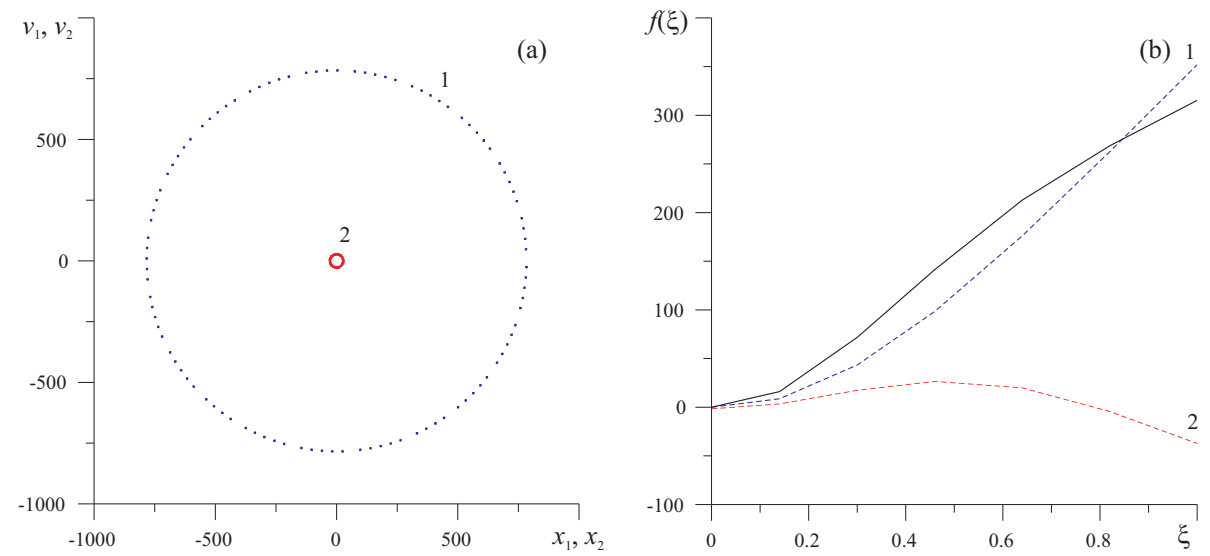

Fig. 4. (a) Typical limit cycles. (1) is the limit cycle displayed by the dynamical variables $\left(x_{1, n}, v_{1, n}\right)$ that correspond to the first spatial mode, (2) is the limit cycle displayed by the second mode. (b) A typical profile of the beam as a function of the longitudinal coordinate $\xi$; The dashed line with mark "1" shows the profile that correspond to the first spatial mode; the dashed line with mark "2" shows the profile that correspond to the second spatial mode. The actual profile of the beam as the superposition of the two modes is illustrated by the black solid line. $\gamma=0.005$ and $f_{1}=0.19$.

\section{ITERATIVE SYSTEM FOR THE PDO}

Since the examined system is subjected to pulsed excitation, between two sampling instants the resonator moves freely, and at each sampling event its velocity is instantaneously changed by the applied delta-pulse. If we define the sequence $\left\{x_{i, n}, \dot{x}_{i, n}\right\}=\left\{x_{i}\left(\tau_{n}\right), d x_{i}\left(\tau_{n}\right) / d \tau\right\}$, that is the sequence of the sampled 'position' and 'velocity' taken from the massspring-damper equations (6) for each $i$ th spatial mode, we will be able to formulate iterative equations that describe the dynamics of the system in terms of the introduced variables [6], [11].

The set of iterative equations has the following form:

$$
\begin{aligned}
& \left(\begin{array}{c}
x_{i, n+1} \\
v_{i, n+1}
\end{array}\right)= \\
& =\alpha_{i}\left(\begin{array}{cc}
\cos 2 \pi f_{i} & -\sin 2 \pi f_{i} \\
\sin 2 \pi f_{i} & \cos 2 \pi f_{i}
\end{array}\right)\left(\begin{array}{c}
x_{i, n} \\
v_{i, n}
\end{array}\right)+\left(\begin{array}{c}
0 \\
\zeta_{i}
\end{array}\right) b_{n-L},
\end{aligned}
$$

where $v_{i, n}=-\left(\rho_{i} x_{i, n}+\dot{x}_{i, n} / \Omega_{i}^{2}\right) /\left(\sqrt{1-\rho_{i}^{2}}\right)$, and $i=$ $\overline{1, M}$. In the set (13), we introduced the following dimensionless parameters:

$$
\begin{array}{rlrl}
\rho_{i} & =\frac{\gamma}{2 \Omega_{i}^{2}}, & f_{i} & =\frac{\Omega_{i}^{2}}{f_{s}} \sqrt{1-\rho_{i}^{2}}, \\
\zeta_{i}=\frac{\sigma F_{0 i}}{\Omega_{i}^{2} \sqrt{1-\rho_{i}^{2}}}, & \alpha_{i} & =\exp \left(\frac{-2 \pi \rho_{i} f_{i}}{\sqrt{1-\rho_{i}^{2}}}\right),
\end{array}
$$

where $\rho_{i}$ is the dimensionless damping parameter, $f_{i}$ is the normalised sample ratio in terms of the paper [6] (or normalised frequency in terms of the paper [11], we will use both terms throughout the paper), $\zeta_{i}$ is the normalised increment (note that it depends on the sign of the feedback loop $\sigma$ ) and $\alpha_{i}$ is the contraction factor. In (13), the first letter of the index, i.e. ' $i$ ', refers to the number of a spatial mode, and the second letter, i.e. ' $n$ ', refers to the iteration number.
Let us introduce the ratio of the spatial frequencies of the modes as follows:

$$
\nu=\frac{\Omega_{2}}{\Omega_{1}},
$$

and apply it to connect the set of parameters for the modes:

$$
\begin{aligned}
\rho_{2}=\frac{\rho_{1}}{\nu^{2}}, & f_{2} \approx \nu^{2} f_{1}, \\
\zeta_{2} \approx \frac{\mu \zeta_{1}}{\nu^{2}}, & \alpha_{2} \approx \alpha_{1} .
\end{aligned}
$$

Note that the parameters that we use for formulation of the iterative system cannot be considered as independent ones, i.e. we have to change the values of controlling parameters according the expressions (16). For instance, in experiments the sampling frequency is measured with respect to the frequency of the fundamental mode (i.e. the first spatial mode with the lowest frequency), thus, the sampling ratio $f_{1}$ is given as a 'basic' parameter. The value of $f_{2}$ can be obtained simply by re-calculating $f_{1}$ with the ratio $\nu$.

The system (13) contains two coupled subsets - the iterative equations that describe the first spatial mode component by the variables $x_{1, n}$ and $v_{1, n}$ and the second spatial mode by the variables $x_{2, n}$ and $v_{2, n}$. Later in the paper, for dynamics that are displayed by the first subset of (13) we will refer to the 'first spatial mode' (or the first mode, eigenmode or resonance), and for dynamics that are displayed by the second subset we will refer to the 'second spatial mode' (or the second mode). Note that the real position of the system is defined by the superposition of these two components according to the formula (4).

\section{RESULTS OF NUMERICAL SIMULATIONS}

In this section we focus on the simplest case of the only two spatial modes with the lowest frequencies taken into consideration. Let us restrict ourselves to the case of the oversampling region, i.e., $0<f_{1}<0.5$. Note that the size of a limit cycle is defined by the normalised increment $\zeta_{i}$ given by the formulas (14) that, in its turn, depends on the amplitude 

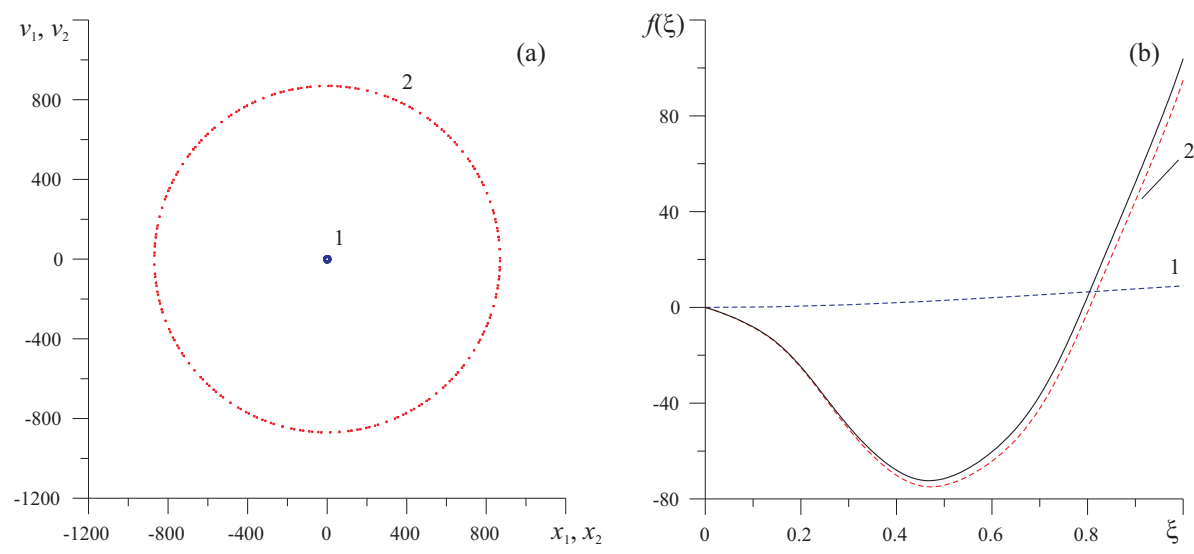

Fig. 5. (a) Typical limit cycles. (1) is the limit cycle displayed by the dynamical variables $\left(x_{1, n}, v_{1, n}\right)$ that correspond to the first spatial mode, (2) is the limit cycle displayed by the second mode. (b) A typical profile of the beam as a function of the longitudinal coordinate $\xi$; The dashed line with mark " 1 " shows the profile that correspond to the first spatial mode; the dashed line with mark " 2 " shows the profile that correspond to the second spatial mode. The actual profile of the beam as the superposition of the two modes is illustrated by the black solid line. $\gamma=0.005$ and $f_{1}=0.024$.

of the external driving, the coefficients (11) and the temporal frequency $\Omega_{i}^{2}$. Thus, in the given range of the sampling ratio and at the chosen values of the coefficients $\beta, \mu$ and $\sigma$, the first spatial mode receives a larger 'portion' of the external driving and should be the predominant one.

Now let us give examples of the system dynamics in terms of the first and the second spatial modes. The results presented below were obtained by iterating the system of equation (13). Figure 4(a) shows typical limit cycles displayed by the variables $x_{1, n}, v_{1, n}$ (the limit cycle denoted as " 1 ") and $x_{2, n}, v_{2, n}$ (the limit cycle denoted as " 2 "). The profile of the beam is given in Fig. 4(b). The dashed lines show the corresponded profiles of the first and the second eigenmode and the solid line illustrates the actual profile of the beam that is formed as the superposition of the modes. The amplitude of the second mode is smaller than the amplitude of the first one, however it affects the overall profile of the cantilever. The values of parameters for the example are given in the figure caption.

However, at high values of the sampling frequency, one can find dominance of the second mode, as shown in Fig. 5. As can be seen, in this case the amplitude of the first spatial mode is smaller than that of the second one (see the limit cycles in Fig. 5(a)) Note that normally the amplitude of suppressed mode is significantly smaller than the amplitude of the dominating one, as is demonstrated in Fig. 5(a). The actual profile of the cantilever corresponds to the second mode and the distortion of the profile caused by the first one is negligible (see Fig. 5(b)).

\section{CONCLusions}

In this paper we have developed a theory that can demonstrate the excitation of various spatial modes in the Pulsed Digital Oscillator for MEMS application. In order to obtain a system of mass-spring-damper equations that defines the dynamics of the spatial modes, we have applied the orderreduction procedure to a partial differential equation that describes the transversal deflections of a beam. Due to the pulsed nature of the external excitation, we have formulated a set of iterative maps to describe the evolution of the modes between two sampling events. We have presented the results of numerical simulations in terms of the spacial modes of the system. Depending on values of the sampling frequency, the system displays oscillations that can be associated with various spatial modes.

\section{REFERENCES}

[1] R. Legtenberg and H. A. C. Tilmans, "Electrostatically driven vacuumencapsulated polysilicon resonators. Part I. Design and fabrication," Sensors and Actuators A, vol. 45, pp. 57-66, 1994.

[2] A. F. Marquès, R. C. Castello, and A. M. Shkel, "Modelling the electrostatic actuation of MEMS: state of the art 2005," Universitat Politècnica de Catalunya, Spain, Tech. Rep. IOC-DT-P-2005-18, Sept. 2005.

[3] V. Cimalla, F. Niebelschütz, K. Tonisch, C. Foerster, K. Brueckner, I. Cimalla, T. Friedrich, J. Pezoldt, R. Stephan, M. Hein, and O. Ambacher, "Nanoelectromechanical devices for sensing applications," Sensors and Actuators B, vol. 126, pp. 24-34, 2007.

[4] G. K.Fedder and R. T. Howe, "Multimode digital control of a suspended polysilicon microstructure," J. Microelectromech. Syst., vol. 5, pp. 283297, Dec. 1996.

[5] M. Domínguez, J. Pons-Nin, J. Ricart, A. Bermejo, E. F. Costa, and M. Morata, "Analysis of the $\Sigma-\Delta$ pulsed digital oscillator for MEMS," IEEE Trans. Circuits Syst. I, vol. 52, pp. 2286-2297, Nov. 2005.

[6] A. Teplinsky and O. Feely, "Limit cycles in a MEMS oscillator," IEEE Trans. Circuits Syst. II, vol. 55, pp. 882-886, Sept. 2008.

[7] E. Blokhina and O. Feely, "A kicked oscillator as a model of MEMS," International Journal of Bifurcations and Chaos, vol. 19, no. 1, p. in press, 2009.

[8] Y. C. Liang and W. Z. Ling, "Proper orthogonal decomposition and its applications - part II: Model reduction for MEMS dynamical analysis," Journal of Sound and Vibration, vol. 256, pp. 515-532, 2002.

[9] A. H. Nayfeh, M. I. Younis, and E. M. Abdel-Rahman, "Reduced-order models for MEMS applications," Nonlinear Dynamics, vol. 41, pp. 211236, 2005.

[10] K. Graff, Wave Motion in Elastic Solids. New York: Dover Publications Inc, 1975.

[11] M. Domínguez, J. Pons-Nin, and J. Ricart, "General dynamics of pulsed digital oscillators," IEEE Trans. Circuits Syst. I, vol. 55, pp. 2038-2050, Aug. 2008.

[12] - "Application of pulsed digital oscillators in 'reverse mode' to eliminate undesired vibrations in high-Q MEMS resonators," in Proc. IEEE International Symposium on Circuits and Systems 2007, New Orleans, USA, May 27-30, 2007, pp. 925-928. 